\title{
Bonificaciones en especie y su efecto en la situación financiera de distribuidores de marca autorizados en el rubro de productos alimenticios
}

\author{
Bonuses in kind and their effect on the financial situation of authorized \\ brand distributors in food products area
}

Roxana Chirinos Baspineiro

roxana.chirinos.b@gmail.com https://orcid.org/0000-0003-0465-8320

Universidad Andina Simón Bolívar, Sucre - Bolivia

Palabras clave

El objetivo de la investigación fue determinar el efecto directo de las bonificaciones en especie en la situación financiera de distribuidores de marca autorizados en el rubro de productos alimenticios. El estudio tuvo dos etapas: primero, se aplicaron entrevistas a profundidad a dueños de diez empresas, para explorar sobre estilos y prácticas de gestión de compras e inventarios, y conocer así la problemática; segundo, se tomaron los estados financieros de una empresa para analizar el efecto directo de las bonificaciones en especie en los indicadores de liquidez, actividad y rentabilidad. Como resultado se determinó que las bonificaciones en especie tienen un impacto negativo en la salud financiera de la empresa estudiada, porque sus decisiones de compra no son estratégicas. Se concluyó que las empresas afectan su rentabilidad por causa de deficiente planificación estratégica en el área, poniendo en riesgo además el cumplimiento de responsabilidades de corto plazo.

Bonificaciones; Mercancías; Rentabilidad; Liquidez; Inventarios

ABSTRACT

Keywords
The objective of the investigation was to determine the direct effect of bonuses in kind on the financial situation of authorized brand distributors in the food products category. The study had two stages: first, in-depth interviews were applied to owners of ten companies, to explore styles and practices of purchasing and inventory management, and thus learn about the problem; second, the financial statements of a company were taken to analyze the direct effect of bonuses in kind on the indicators of liquidity, activity, and profitability. As a result, it was determined that bonuses in kind or negatively affect the financial health of the company studied, because its purchase decisions are not strategic. It was concluded that companies prove their profitability due to deficient strategic planning in the area, also putting at risk the fulfillment of short-term responsibility.

Bonuses; Goods; Cost effectiveness; Liquidity; Inventories 


\section{INTRODUCCIÓN}

Las empresas industriales, en el afán de lograr mayores ventas, otorgan a sus distribuidores autorizados bonificaciones en especie por el cumplimiento de metas en compras, impuestas por ellos mismos, que implica que el distribuidor que realiza compras elevadas de mercadería recibe como premio mercadería sin costo adicional, como bonificaciones (Acevedo \& Gómez, 2001; Jaramillo \& Bohórquez, 2008). Esta estrategia puede ser perjudicial para las empresas que carecen de planificación estratégica en el área de adquisiciones, ya que crea la ilusión de un beneficio que en realidad genera acumulación de inventarios y problemas posteriores (Rizzo \& Vélez, 2018; Ballou, 2004; Castillo, 2005). En este sentido, la gestión de inventarios es un tema de vital importancia en las empresas comerciales, la falta de una planificación estratégica en el proceso de compras de mercaderías conlleva consecuencias que pueden afectar los rendimientos esperados en la empresa (Eppen et al., 2000; Ballou, 2004; Ferrín, 2007).

Las medianas y pequeñas empresas (MyPEs) de este rubro, que no tienen una administración eficiente, presentan problemas en el manejo de inventarios (González et al., 2002; Muller, 2005; Perdomo, 2004). En el contexto boliviano, la generalidad de los distribuidores de marca autorizados, es decir más del $50 \%$, son MyPEs, e inclusive empresas unipersonales. Éstas no necesariamente realizan planificación y controles rigurosos de inventarios. En este sentido, frente a los incentivos antes expuestos, realizan compras de los productos sin respaldo en un análisis previo de inventarios. Esto provoca una fuerte inversión en mercadería que permanece por tiempos largos en almacenes. Adicionalmente, se recibe la bonificación que también se encuentra en stock. Al no existir acciones estratégicas que repercutan en el incremento en las ventas, se generan costos adicionales por el almacenamiento de mercadería.

En Sucre, ciudad capital del departamento de Chuquisaca, existen alrededor de 200 empresas distribuidoras autorizadas de marcas del rubro de productos alimenticios. Éstas tienen problemas constantes en el manejo de inventarios, como consecuencia de deficiente gestión interna. Ante esto, las comprar mayores que realizan para obtener premios y bonificaciones, agravan los problemas en almacenes de mercaderías y de sobreinversión en la cuenta de inventarios. En el marco de esta problemática, el objetivo del artículo fue analizar el efecto directo de las bonificaciones en especie en la situación financiera de los distribuidores de marca autorizados en el rubro de productos alimenticios.

\section{METODOLOGÍA}

La investigación fue desarrollada bajo un enfoque positivista, en dos etapas. La primera se caracterizó por ser de alcance exploratorio; la información que se presenta como fruto de esta primera etapa fue obtenida a través de entrevistas a 10 dueños de empresas distribuidoras autorizadas de marcas del rubro de productos alimenticios, se utilizaron como instrumentos dos tipos de cuestionarios. El primero conformado por ocho ítems referidos a conocer información general de la empresa (dirección, tipo de constitución, tamaño y estructura organizacional, productos que distribuyen, estilo de dirección). El segundo cuestionario estuvo orientado a obtener información sobre el manejo de los inventarios, compras de la empresa y acciones de planificación y dirección estratégica. Ambos instrumentos fueron validados mediante criterios de expertos, la selección de la muestra fue no probabilística por conveniencia.

ISSN: 2788 - 6557| ISNN-L: 2788 - 6557
Volumen 3 | No. 2 | Julio - diciembre 2021
www.revistapanel.org


La segunda atapa se caracterizó por aplicar técnicas cuantitativas y alcance descriptivo. Se tomó como caso de estudio a una empresa dedicada a la distribución de la marca Stege en la ciudad de Sucre (Bolivia). La empresa desarrolla actividades desde la gestión 2006, sin embargo, por las características del estudio solamente fue necesario tomar en análisis las dos últimas gestiones (gestión 1 = año 2018 y gestión 2 = año 2019). A partir de ello, se analizaron los estados financieros (balancegeneralyestado de resultados) de dos gestiones consecutivas y en dos momentos: se partió por el análisis de los estados financieros con los datos registrados en la contabilidad de la empresa: no se registra la partida bonificaciones, a pesar de recibir permanentemente bonificaciones en especie como premio por compras mayores; posteriormente se procedió a analizar los estados financieros "corregidos". Finalmente, se contrastaron los indicadores de liquidez, actividad y rentabilidad.

La base teórica del estudio ha sido recogida de: Gallagher y Watson (2005), Gil (2004), Eppen et al. (2000) y Ferrín (2007).

\section{RESULTADOS}

Los resultados del estudio se presentan en dos apartados. Primero, se analiza la problemática entorno a las compras con cumplimiento de metas realizadas por los distribuidores de marca autorizados en el rubro de productos alimenticios, y las consecuencias que los representantes advierten. Segundo, mediante un caso de estudio, se mide el efecto de las bonificaciones sobre compras en los indicadores de liquidez, actividad y rentabilidad.

La información que se presenta a continuación fue obtenida a través de entrevistas a los representantes de estas distribuidoras. La indagación estuvo orientada a obtener información sobre el manejo de los inventarios y compras de la empresa y sobre la planificación estratégica relacionada con compras.

\section{Manejo de los inventarios y compras de la empresa}

Se evidenció lo siguiente: [1] En general, los distribuidores no tienen definido un método de valuación de sus inventarios, por lo tanto, carecen de información a tiempo real del costo de cada producto; [2] El 80\% carece de controles sistemáticos en el manejo de la mercadería, son los dueños los responsables de adquirir, recibir, almacenar y pagar por la mercadería (trabajan con contador externo). El restante $20 \%$ realiza un control sistemático y consideran las bonificaciones que el proveedor entrega por compras por cumplimiento de ventas (físicamente en almacenes y contablemente como bonificaciones); [4] El $80 \%$ de las empresas consultadas no realiza un prorrateo que incluya la bonificación para determinar el costo unitario real de las compras por cumplimiento de metas; [5] El $90 \%$ de las empresas no realizan devoluciones sobre ventas, por lo tanto, no existe ningún registro de ello; el $100 \%$ registran las devoluciones sobre compras.

Analizando exclusivamente los casos de las empresas que carecen de controles sistemáticos en el manejo de la mercadería ( $80 \%$ del total) se afirma lo siguiente: Los incentivos o premios en compras no están debidamente registrados, ni valuados; en estos casos se carece de registros auxiliares de la mercadería que entra a la empresa y por consiguiente no se puede determinar si hubo diferencias. Al no tener un correcto registro de la mercadería ingresada, tampoco se puede registrar ajustes de ella.

Con relación a las empresas proveedoras de mercaderías, éstas fijan los precios de venta de los productos, lo que indica que los precios se van actualizando en relación con los costos directos. 


\section{Planificación estratégica relacionada y políticas de compras}

En términos de planificación estratégica, ninguna de las empresas desarrolla una planificación de las compras que realizará en el año. El $70 \%$ de las empresas afirma que realiza inventarios físicos anualmente debido, principalmente, al poco tiempo que disponen por la carga laboral del negocio. En el $70 \%$ de las empresas, las compras se realizan según criterio de los dueños o gerentes, no se cuenta con análisis histórico ni con proyecciones de ventas.

En general, los entrevistados detectan problemas por incorrectos cálculos en los manejos de inventarios; periódicamente enfrentan pérdidas por vencimientos de productos; los almacenes rebasan su capacidad máxima; falta de liquidez para enfrentar las obligaciones de corto plazo.

Entre las acciones estratégicas relacionadas, los propietarios destacan las compras con cumplimiento de metas y las compras a crédito. Al respecto corresponde aclarar que, compras por cumplimiento de metas es una modalidad en la que el proveedor otorga bonificaciones en especie a las empresas que realizan pedidos mayores, de esta manera el comprador recibe una cantidad extra del mismo producto. El beneficio implícito está en que el costo unitario de esta mercancía baja, sin embargo, como se explicó previamente, la generalidad de estas empresas no registra el prorrateo del costo en sus estados financieros. La otra acción estratégica que fue mencionada es la compra a crédito, que según se evidenció, provoca de alguna manera problemas de liquidez.

Los entrevistados hacen énfasis en la complejidad del manejo de mercaderías en el rubo de alimentos, por la fecha de caducidad y las especificaciones ambientales para transporte y almacenamiento.

\section{Efecto de bonificaciones en estados financieros: caso de estudio}

El sujeto de investigación en esta etapa fue una empresa que desde 2006 se dedica a la distribución de la marca Stege en la ciudad de Sucre. Los productos que distribuye son escabeches, embutidos y carnes frias.

Debido al éxito y aceptación de los productos edurante los diez primeros años de actividad en Sucre, la distribuidora empezó a proveerse de mercadería en cantidades mayores y a la vez a acceder a incentivos o premios ofrecidos por el proveedor, pero se identificó desproporción entre ingresos y salidas de inventarios. Esto empezó a generar problemas de liquidez, costos adicionales de almacenaje, control de los inventarios y otros.

A partir de esta situación problamética, se analizaron los estados financieros de dos gestiones consecutivas y en dos momentos: se partió por el análisis de los estados financieros con los datos registrados en la contabilidad de la empresa (nótese que esta distribuidora no registra la partida bonificaciones, que recibe por compras por cumplimiento de metas); posteriormente se procedió a analizar los estados financieros "corregidos", es decir, considerando las bonificaciones que el proveedor entrega por compras mayores (contablemente). Finalmente, se compararon los indicadores de liquidez, actividad y rentabilidad.

Se recabaron los estados financieros de las gestiones 2018 y 2019, de aquí en adelante denominadas gestión 1 y gestión 2. En estos documentos se observa que no están registradas las bonificaciones sobre compras. En la Tabla 1 se presenta el balance general de la empresa (gestiones 1 y 2 ). 
Tabla 1. Balance general (en BOB).

\begin{tabular}{|c|c|c|}
\hline & Año 2 & Año 1 \\
\hline ACTIVO CORRIENTE & $519.673,39$ & $324.531,05$ \\
\hline Disponible: & $104.751,34$ & $78.423,00$ \\
\hline Banco $\mathrm{M} / \mathrm{N}$ & $104.751,34$ & $78.423,00$ \\
\hline Exigible: & $166.725,05$ & $177.608,05$ \\
\hline Cuentas por cobrar & $150.000,00$ & $150.000,00$ \\
\hline IUE por compensar & $16.725,05$ & $27.608,05$ \\
\hline Realizable & $248.197,00$ & $68.500,00$ \\
\hline Inv. De Mercaderías & $248.197,00$ & $68.500,00$ \\
\hline ACTIVO NO CORRIENTE & $31.825,41$ & $125.560,91$ \\
\hline Bienes de Uso: & $31.825,41$ & $125.560,91$ \\
\hline Muebles y Enseres & $24.675,55$ & $23.838,38$ \\
\hline (-) Deprec. Acum. Muebles y Enseres & $21.691,71$ & $18.571,93$ \\
\hline Equipos de computación & $18.520,45$ & $17.892,09$ \\
\hline (-) Deprec. Acum. Equipo de Computación & $11.189,43$ & $6.336,78$ \\
\hline Vehículos & $455.236,87$ & $439.791,94$ \\
\hline (-) Deprec. Acum. Vehículos & $433.726,32$ & $331.052,79$ \\
\hline TOTAL ACTIVO & $551.498,80$ & $450.091,96$ \\
\hline PASIVOS CORRIENTE & $277.099,37$ & $159.349,05$ \\
\hline Cuentas por pagar & - & $111.385,00$ \\
\hline IVA por pagar & $141.803,00$ & $13.188,00$ \\
\hline IUE por pagar & $15.947,00$ & $27.608,05$ \\
\hline IT por pagar & $53.772,86$ & $7.168,00$ \\
\hline Aportes patronales por pagar & $3.770,19$ & - \\
\hline Caja de Salud por pagar & $5.618,76$ & - \\
\hline Aportes laborales por pagar & $7.141,44$ & - \\
\hline Sueldos y salarios por pagar & $49.046,12$ & - \\
\hline PASIVOS NO CORRIENTE & - & $60.000,00$ \\
\hline Documento por pagar & - & $60.000,00$ \\
\hline PATRIMONIO & $274.399,43$ & $230.742,91$ \\
\hline Capital Propio & $37.618,45$ & $37.618,45$ \\
\hline Ajuste de Capital & & $22.884,43$ \\
\hline Ajuste de Reservas Patrimoniales & & $8.852,69$ \\
\hline Resultados Acumulados & & $121.403,89$ \\
\hline Resultados de la gestión & & $39.983,45$ \\
\hline TOTAL PASIVO Y PATRIMONIO & $551.498,80$ & $450.091,96$ \\
\hline
\end{tabular}


En la Tabla 2 se presenta el estado de resultados para las gestiones 1 y 2 . Según los datos contabilizados por la empresa; le empresa no registra las bonificaciones.

Tabla 2. Estado de resultados (en BOB).

\begin{tabular}{|c|c|c|}
\hline & Año 2 & Año 1 \\
\hline \multicolumn{3}{|l|}{ INGRESOS } \\
\hline Ingresos por ventas & 2.149.828,59 & $1.912 .908,30$ \\
\hline Ventas & $2.110 .151,94$ & $1.881 .607,29$ \\
\hline Ventas Re expresadas & $39.676,65$ & $31.301,01$ \\
\hline COSTO DE MERCADERÍAS VENDIDAS & $810.196,17$ & $1.387 .839,47$ \\
\hline Inv. Inicial & $68.500,00$ & $26.450,00$ \\
\hline Compras & $1.335 .083,94$ & $1.634 .369,47$ \\
\hline (-) Devoluciones sobre compras & $120.311,43$ & $144.823,00$ \\
\hline (-) Descuentos sobre compras & $224.879,34$ & $59.657,00$ \\
\hline Inv. Disponible para la venta & $1.058 .393,17$ & $1.456 .339,47$ \\
\hline Inv. Final & $248.197,00$ & $68.500,00$ \\
\hline RESULTADOS BRUTOS EN VENTAS & $1.339 .632,42$ & $525.068,83$ \\
\hline \multicolumn{3}{|l|}{ GASTOS DE OPERACIÓN } \\
\hline Gastos de Administración & $1.064 .888,71$ & $308.200,86$ \\
\hline Impuestos & $130.371,15$ & $65.944,69$ \\
\hline Impuesto a las transacciones & $72.763,86$ & $65.944,69$ \\
\hline Impuesto a los consumos específicos & $52.259,29$ & - \\
\hline Impuesto a las utilidades de las empresas & $5.348,00$ & - \\
\hline Reposición de Bienes de Uso & $98.145,04$ & $92.578,74$ \\
\hline Deprec. Muebles y Enseres & $2.467,56$ & - \\
\hline Deprec. Equipo de Computación & $4.630,11$ & - \\
\hline Deprec. Vehículos & $91.047,37$ & \\
\hline RESULTADOS EN OPERACIONES & $46.227,52$ & $58.344,54$ \\
\hline OTROS INGRESOS Y EGRESOS & $9.270,23$ & $18.361,09$ \\
\hline Diferencia por redondeo & 1,77 & - \\
\hline Re-expresión de Egresos & & $27.447,71$ \\
\hline Ajuste por Inflación y Tenencias de Bienes & $9.272,00$ & $9.086,62$ \\
\hline UTLIDAD NETA IMPONIBLE & $36.957,29$ & $39.983,45$ \\
\hline Impuesto a las utilidades de las empresas & $9.239,32$ & $9.995,86$ \\
\hline UTILIDAD NETA & $27.717,97$ & $29.987,59$ \\
\hline
\end{tabular}

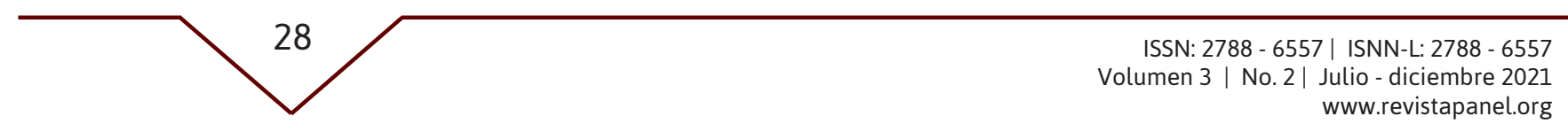


Con base en la información presentada en las Tablas 1 y 2, se analizan los indicadores de liquidez, actividad y rentabilidad.

Tabla 3. Análisis de liquidez, actividad y rentabilidad

\begin{tabular}{cccc}
\hline Indicador/índice & Fórmula & Año 2 & Año 1 \\
\hline Razón corriente & activo corriente / pasivo corriente & 1,88 & 2,04 \\
Prueba ácida & (activo corriente - inventarios) / pasivo corriente & 0,98 & 1,61 \\
Rotación de inventarios & costo de ventas / inventario promedio & 5,12 & 29,23 \\
Rendimiento sobre la inversión (ROI) & (utilidad neta / activo total) $\times 100$ & 0,05 & 0,07 \\
Margen de utilidad & (utilidad neta / ventas netas) *100 & 0,01 & 0,02 \\
Rendimiento del patrimonio (ROE) & (utilidad neta / patrimonio) * 100 & 0,10 & 0,13 \\
\hline
\end{tabular}

Los resultados de la Tabla 3 se interpretan de la siguiente manera.

Índices de liquidez: Razón corriente, la empresa tiene alta capacidad para cubrir deudas adquiridas en el corto plazo, en la gestión 1 tuvo 2,04 BOB de activos corrientes (incluidos inventarios) para cubrir 1 BOB de deuda corriente, en la gestión 2 tuvo menos (1,88 BOB); Prueba ácida, demuestra que la capacidad de la empresa para cubrir sus deudas excluyendo inventarios es menor. Se deduce que la empresa tiene una alta inversión en inventarios que pueden llegar a perjudicar el pago de obligaciones a corto plazo (gestión 2). Asimismo, dentro del activo corriente, en el grupo exigible, está también incluida la cuenta IUE por compensar que, si bien favorece en la compensación de impuestos para cubrir deuda a corto plazo, no podría convertirse en disponible en ninguna circunstancia. La empresa necesariamente depende de sus ventas para cubrir su deuda a corto plazo.

Índice de actividad: Los resultados muestran que los inventarios en la gestión 1 rotaron 29.23 veces, este resultado se interpreta como muy favorable, la empresa fue capaz de generar mayores ventas con mayores márgenes de utilidad. En la gestión 2, los inventarios rotaron 5.12 veces, comparando este resultado con la gestión 1 , ha disminuido sustancialmente, debido a que hubo un incremento en la compra de mercaderías para el cumplimiento de metas y no se generó un incremento de las ventas, lo que hizo también que el inventario final sea elevado.

Índices de rentabilidad: ROI, de toda la inversión realizada, se obtuvo una utilidad neta del 7\% (gestión 1) y 5\% (gestión 2); El margen de utilidad es muy bajo por la alta competencia con otras marcas, la gestión 1 fue $2 \%$ y la gestión 2 se redujo a la mitad; ROE, por cada unidad monetaria que el dueño mantuvo en la empresa en la gestión 1, generó un rendimiento del 13\% sobre su patrimonio, y en la gestión 2 se obtuvo un rendimiento del $10 \%$.

Continuando con el análisis, se "corrigieron" los estados de resultados (Tabla 4) y los balances generales (Tabla 5) de ambas gestiones, incorporando contablemente las bonificaciones que el proveedor entrega por compras mayores. 
Tabla 4. Balance general considerando bonificaciones (en BOB).

\begin{tabular}{|c|c|c|}
\hline & Año 2 & Año 1 \\
\hline ACTIVO CORRIENTE & $1.013 .638,15$ & $945.591,45$ \\
\hline Disponible: & $104.751,34$ & $78.423,00$ \\
\hline Banco M/N & $104.751,34$ & $78.423,00$ \\
\hline Exigible: & $163.263,05$ & $177.608,05$ \\
\hline Cuentas por cobrar & $150.000,00$ & $150.000,00$ \\
\hline IUE por compensar & $13.263,05$ & $27.608,05$ \\
\hline Realizable & $745.623,76$ & $689.560,40$ \\
\hline Inv. De Mercaderías & $745.623,76$ & $689.560,40$ \\
\hline ACTIVO NO CORRIENTE & $31.825,41$ & $125.560,91$ \\
\hline Bienes de Uso: & $31.825,41$ & $125.560,91$ \\
\hline Muebles y Enseres & $24.675,55$ & $23.838,38$ \\
\hline (-) Deprec. Acum. Muebles y Enseres & $21.691,71$ & $18.571,93$ \\
\hline Equipos de computación & $18.520,45$ & $17.892,09$ \\
\hline (-) Deprec. Acum. Equipo de Computación & $11.189,43$ & $6.336,78$ \\
\hline Vehículos & $455.236,87$ & $439.791,94$ \\
\hline (-) Deprec. Acum. Vehículos & $433.726,32$ & $331.052,79$ \\
\hline TOTAL ACTIVO & $1.045 .463,56$ & 1.071.152,36 \\
\hline PASIVOS CORRIENTE & $758.081,29$ & $761.777,64$ \\
\hline Cuentas por pagar & $556.832,09$ & $713.813,59$ \\
\hline IVA por pagar & $69.414,83$ & $13.188,00$ \\
\hline IUE por pagar & $12.485,00$ & $27.608,05$ \\
\hline IT por pagar & $53.772,86$ & $7.168,00$ \\
\hline Aportes patronales por pagar & $3.770,19$ & - \\
\hline Caja de Salud por pagar & $5.618,76$ & - \\
\hline Aportes laborales por pagar & $7.141,44$ & - \\
\hline Sueldos y salarios por pagar & $49.046,12$ & - \\
\hline PASIVOS NO CORRIENTE & - & $60.000,00$ \\
\hline Documento por pagar & - & $60.000,00$ \\
\hline PATRIMONIO & $287.382,27$ & $249.374,72$ \\
\hline Capital Propio & $37.618,45$ & $37.618,45$ \\
\hline Ajuste de Capital & $24.205,54$ & $22.884,43$ \\
\hline Ajuste de Reservas Patrimoniales & $9.967,26$ & $8.852,69$ \\
\hline Resultados Acumulados & $165.650,89$ & $121.403,89$ \\
\hline Resultados de la gestión & $49.940,13$ & $58.615,26$ \\
\hline TOTAL PASIVO Y PATRIMONIO & $1.045 .463,56$ & $1.071 .152,36$ \\
\hline
\end{tabular}

ACTIVO CORRIENTE

Exigible:

Cuentas por cobrar

IUE por compensar

Inv. De Mercaderías

Muebles y Enseres

(-) Deprec. Acum. Muebles y Enseres

(-) Deprec. Acum. Equipo de Computación

TOTAL ACTIVO

PASIVOS CORRIENTE

Cuentas por pagar

IVA por pagar

IT por pagar

Aportes patronales por pagar

Caja de Salud por pagar

Aportes laborales por pagar

PASIVOS NO CORRIENTE

por pagar

Capital Propio

Resultados Acumulados

TOTAL PASIVO Y PATRIMONIO 
En la Tabla 5 se presenta el estado de resultados de las gestiones 1 y 2 con la incorporación de las bonificaciones.

Tabla 5. Estado de resultados considerando bonificaciones (en BOB).

\begin{tabular}{|c|c|c|}
\hline & Año 2 & Año 1 \\
\hline \multicolumn{3}{|l|}{ INGRESOS } \\
\hline Ingresos por ventas & $2.149 .828,59$ & $1.912 .908,30$ \\
\hline Ventas & $2.110 .151,94$ & $1.881 .607,29$ \\
\hline Ventas Re expresadas & $39.676,65$ & $31.301,01$ \\
\hline COSTO DE MERCADERÍAS VENDIDAS & $797.213,33$ & $1.369 .207,66$ \\
\hline Inv. Inicial & $68.500,00$ & $26.450,00$ \\
\hline Compras & $1.832 .510,70$ & $2.255 .429,87$ \\
\hline (-) Devoluciones sobre compras & $120.311,43$ & $144.823,00$ \\
\hline (-) Descuentos sobre compras & $224.879,34$ & $59.657,00$ \\
\hline Inv. Disponible para la venta & $12.982,84$ & $18.631,81$ \\
\hline Inv. Final & $1.542 .837,09$ & $2.058 .768,06$ \\
\hline RESULTADOS BRUTOS EN VENTAS & $745.623,76$ & $689.560,40$ \\
\hline GASTOS DE OPERACIÓN & $1.352 .615,26$ & $543.700,64$ \\
\hline Gastos de Administración & $1.064 .888,71$ & $308.200,86$ \\
\hline Impuestos & $130.371,15$ & $65.944,69$ \\
\hline Impuesto a las transacciones & $72.763,86$ & $65.944,69$ \\
\hline Impuesto a los consumos específicos & $52.259,29$ & - \\
\hline Impuesto a las utilidades de las empresas & $5.348,00$ & - \\
\hline Reposición de Bienes de Uso & $98.145,04$ & $92.578,74$ \\
\hline Deprec. Muebles y Enseres & $2.467,56$ & - \\
\hline Deprec. Equipo de Computación & $4.630,11$ & - \\
\hline Deprec. Vehículos & $91.047,37$ & - \\
\hline RESULTADOS EN OPERACIONES & $59.210,36$ & $76.976,35$ \\
\hline OTROS INGRESOS Y EGRESOS & $9.270,23$ & $18.361,09$ \\
\hline Diferencia por redondeo & 1,77 & - \\
\hline Re-expresión de Egresos & & $27.447,71$ \\
\hline Ajuste por Inflación y Tenencias de Bienes & $9.272,00$ & $9.086,62$ \\
\hline UTLIDAD NETA IMPONIBLE & $49.940,13$ & $58.615,26$ \\
\hline Impuesto a las utilidades de las empresas & $12.485,03$ & $14.653,82$ \\
\hline UTILIDAD NETA & $37.455,10$ & $43.961,45$ \\
\hline
\end{tabular}

Con base en la información presentada en las Tablas 4 y 5, se analizan los indicadores de liquidez, actividad y rentabilidad. Nótese que los datos registran las bonificaciones que la empresa recibió por cumplimiento de metas durante estas dos gestiones, es decir, la interpretación es más apropiada y responde a la actividad real de la empresa. 
Tabla 6. Análisis de liquidez, actividad y rentabilidad (considerando bonificaciones).

\begin{tabular}{cccc}
\hline Indicador/índice & Fórmula & Año 2 & Año $\mathbf{1}$ \\
\hline Razón corriente & activo corriente / pasivo corriente & 1,34 & 1,24 \\
Prueba ácida & (activo corriente - inventarios) / pasivo corriente & 0,35 & 0,34 \\
Rotación de inventarios & costo de ventas / inventario promedio & 1,11 & 3,82 \\
Rendimiento sobre la inversión (ROI) & (utilidad neta / activo total) $\times 100$ & 0,04 & 0,04 \\
Margen de utilidad & (utilidad en operaciones / ventas) ${ }^{*} 100$ & 0,017 & 0,023 \\
Rendimiento del patrimonio (ROE) & (utilidad neta / patrimonio) ${ }^{*} 100$ & 0,13 & 0,18 \\
\hline
\end{tabular}

Los resultados de la Tabla 6 se interpretan de la siguiente manera.

Índices de liquidez: Razón corriente, la empresa tiene capacidad para cubrir deudas adquiridas en el corto plazo, en la gestión 2 tuvo 1,24 BOB de activos corrientes (incluidos inventarios) para cubrir $1 \mathrm{BOB}$ de deuda corriente, en la gestión 1 tuvo 1,34 BOB; La prueba ácida muestra que, incorporando el registro contable de las bonificaciones, la empresa tuvo en las gestiones 1 y 2 solamente 0,34 y 0,35 BOB para cubrir cada 1 BOB de deuda corriente. La empresa tiene problemas de liquidez y necesariamente depende de sus ventas para cubrir deuda a corto plazo.

Índice de actividad: Considerando las características de los productos que oferta la empresa y en especial tomando en cuenta que se trata de productos alimenticios, se afirma que la rotación de inventarios en ambas gestiones es muy baja.

Índices de rentabilidad: ROI, en ambas gestiones el rendimiento sobre la inversión es del $4 \%$, inferior al registrado en la Tabla 3 (sobre todo en la gestión 1); El margen de utilidad es ligeramente más bajo al obtenido según la contabilidad que realiza la empresa regularmente; según el ROE, por cada unidad monetaria que el dueño mantuvo en la empresa en la gestión 1 , generó un rendimiento del $18 \%$ sobre su patrimonio, y en la gestión 2 se obtuvo un rendimiento del $13 \%$.

Tabla 7. Análisis de liquidez, actividad y rentabilidad (comparativo, con y sin bonificaciones).

\begin{tabular}{|c|c|c|c|c|}
\hline \multirow{3}{*}{ Indicador/índice } & \multirow{2}{*}{\multicolumn{2}{|c|}{ Según registros de la empresa }} & \multirow{2}{*}{\multicolumn{2}{|c|}{$\frac{\text { Corregido }}{\text { (incorporando bonificaciones) }}$}} \\
\hline & & & & \\
\hline & Año 1 & Año 2 & Año 1 & Año 2 \\
\hline Razón corriente & 1,88 & 2,04 & 1,34 & 1,24 \\
\hline Prueba ácida & 0,98 & 1,61 & 0,35 & 0,34 \\
\hline Rotación de inventarios & 5,12 & 29,23 & 1,11 & 3,82 \\
\hline Rendimiento sobre la inversión (ROI) & 0,05 & 0,07 & 0,04 & 0,04 \\
\hline Margen de utilidad & 0,01 & 0,02 & 0,017 & 0,023 \\
\hline Rendimiento del patrimonio (ROE) & 0,10 & 0,13 & 0,13 & 0,18 \\
\hline
\end{tabular}


En la Tabla 7 se observa que por el incremento en compras y el registro de las bonificaciones en especie hay un decremento en los resultados del 0.54 en la gestión 2, y de 0.80 en la gestión 1. Esta disminución de los índices se debe a que el pasivo corriente se incrementa, al incrementarse las compras de mercadería, además que se incluye en el registro las bonificaciones sobre compras. Si bien al incrementar las compras se recibe un incentivo que es la bonificación en especie que va a disminuir el costo de adquisición, tiene un efecto negativo ya que dichas compras se las hacen a crédito, lo que aumenta el pasivo corriente.

Al comparar los resultados de una gestión a otra, hay un decremento en los índices, debido al incremento en el pasivo corriente por la obligación contraída derivada del aumento en compras. En los dos análisis, ya sea con los datos reales o con considerando las bonificaciones, la empresa no es capaz de cubrir sus obligaciones a corto plazo sin tener que realizar sus inventarios; el aumento en compras no es favorable cuando la empresa adquiere obligaciones por pagar a corto plazo derivadas de dichas compras.

En relación con la rotación de inventarios, se observa una disminución del $1 \%$ en la gestión 2 y del $3 \%$ en la gestión 1 . Al considerar este índice en el análisis se pretender mostrar que, si bien las compras con incentivos parecen que darán un beneficio extra, en realidad hacen que la empresa llegue a acumular mercadería en almacenes. Si la empresa paralelamente no incrementa sus ventas, lo que logrará es que haya una baja rotación de inventarios como se muestra en la Tabla 7; en corrección no se afectó la partida de ingresos para mostrar el comportamiento de los estados financieros con una acumulación de mercadería a consecuencia de las compras y de la mercadería extra que llega como bonificación.

Los resultados del rendimiento sobre la inversión muestran una disminución en la gestión 2 del $1 \%$ y en la gestión 1 del 3\%. Aunque la utilidad neta se incrementa en el segundo análisis, debido a las bonificaciones en especie, el activo total también se incrementa por las existencias en inventarios y esto es lo que provoca la disminución en el índice. Por consiguiente, no es suficiente que se obtenga la mercadería a un menor precio como sucede al recibir la bonificación en especie, sino que la empresa debe considerar otros aspectos como las ventas, la disminución de costos de almacenaje, costo del pedido, transporte de la mercadería y la disminución de gastos operativos, para tener un mejor rendimiento sobre la inversión.

Contrariamente a los anteriores índices, en el margen neto de utilidad, se muestra resultados positivos con el incremento en las compras y el registro de las bonificaciones, esto se debe a que las variables que considera el índice son variables del estado de resultados, que no están influidas por otros aspectos que se presentan en el balance general. Independientemente de lo mencionado en el párrafo anterior, se puede decir que los márgenes de utilidad de la empresa son relativamente bajos. Al analizar solo el estado de resultados, se ve que al incrementar las compras y acceder a la bonificación en especie, existe un beneficio ya que la utilidad neta también se incrementa de 36957.29 BOB a 49940.13 BOB. En la gestión 2, y en la gestión 1 de 39983.45 BOB a 58615.26 BOB. Este incremento en la utilidad neta proviene de las bonificaciones en especie, ya que estas al registrarse, disminuyen el costo de adquisición y por ende el costo de ventas.

\section{CONCLUSIONES}

Entre las gestiones de análisis 1 y 2 la empresa tuvo un crecimiento considerable atribuible, principalmente, a la buena aceptación del producto en el mercado local, mismo que se ve perjudicado por la falta de planificación y un gestión de inventarios que carece de control riguroso. De la misma manera, se advierte necesidad primordial en implementar prácticas de 
planificación respecto a compras de mercadería para evitar sobre acumulación y sobreinversión que afecte la liquidez de la empresa. La empresa depende necesariamente del incremento en venta de productos para conseguir equilibrio entre la inversión en inventarios, mejores rendimientos y liquidez, que posibilite el cumplimiento de sus obligaciones a corto plazo.

Asimismo, tiene un margen de utilidad muy bajo (inferior a la tasa pasiva otorgada a depósitos a plazo fijo), ya que el producto que se oferta es de consumo masivo y se comercializa en un mercado altamente competitivo, esto permite tener márgenes de utilidad reducidos. Los rendimientos sobre la inversión son relativamente bajos, esto es parcialmente causa del excesivo gasto de operaciones que tiene la empresa, que hace que la utilidad neta se vea disminuida.

Tomando en cuenta los análisis realizados, se concluye que la empresa no se encuentra en una situación financiera saludable, su pasivo superaba el patrimonio. A pesar de ello se hizo el análisis de índices correspondientes para determinar si realmente las compras realizadas para hacerse acreedor de incentivos o bonificaciones en especie, beneficiaba o no a la empresa. Se puede decir entonces que considerar las bonificaciones sobre compras tienen un efecto positivo, al ser registrada la mercadería como bonificación provoca que el costo de adquisición y de ventas de los productos bajen y por consiguiente suba la utilidad neta. Sin embargo, se debe considerar que realizar compras mayores con bonificaciones puede generar pasivos a corto plazo que, de no lograr un incremento planificado en ventas, derivarán en problemas de liquidez.
REFERENCIAS

Acevedo, J. y Gómez, M. (2001) Gestión de Inventarios, Habana: Editorial ISPJAE

Ballou, R. H. (2004) Logística: administración de la cadena de suministro ( $5^{a}$. Ed.). México: Pearson Prentice Hall

Castillo, G. (2005). Propuesta de política de inventarios para productos " $A$ " de la empresa REFA Mexicana S.A. de C.V., Tesis. Universidad de las Américas, Puebla

Eppen, G.D., Gould, F. J., Schmidt, C.P., Moore, J. H. y Weatherford, L. R. (2000) Investigación de operaciones en la ciencia administrativa. México: Pearson Prentice Hall

Ferrín, G. A. (2007) Gestión de stocks en la logística de almacenes. Madrid: Fund. Confemetal Editorial

Gallagher, Ch. A. y Watson, H. J. (2005) Métodos cuantitativos para la toma de decisiones en administración. México: McGraw-Hill

Gil, A. A. (2004) Introducción al análisis financiero ( $3^{\text {a }}$. ed.) España: Editorial Club Universitario

González, G. J. I., Morini, M. S. y Do Nascimento, E. (2002) Control y gestión del área comercial y de producción de la PYME. España: Netbiblo

Jaramillo, G. J. S., \& Bohórquez, J. A. R. (2008). La motivación en equipos de ventas. Directivos Universidad EAFIT, 31

Muller, M. (2005) Fundamentos de administración de inventarios. Colombia: Editorial Norma.

Perdomo, M. A. (2004) Fundamentos de control interno. México: Cengage Learning Editores

Rizzo Mendoza, A. A., \& Vélez Montesdeoca, J. D. (2018). Análisis y optimización de procesos de inventario en las empresas textiles de Guayaquil. Caso de estudio Distribuidora Esprit SA (Bachelor's thesis, Universidad de Guayaquil Facutad de Ciencias Administrativas) 